Article

\title{
Multiband SHEPWM Control Technology Based on Walsh Functions
}

\author{
Song Li $₫$, Guizhi Song, Manyuan Ye *, Wei Ren and Qiwen Wei
}

College of Electrical and Automation Engineering, East China Jiaotong University, Nanchang 330013, China; jxlisong1@163.com (S.L.); SGZ_318@163.com (G.S.); renwei_1995@163.com (W.R.); weiqiwens@163.com (Q.W.)

* Correspondence: myye@ecjtu.edu.cn

Received: 7 May 2020; Accepted: 10 June 2020; Published: 15 June 2020

\begin{abstract}
The drawback of modulation ratio limitation in selective harmonic elimination pulse width modulation (SHEPWM) technology based on Walsh function transformation. In order to solve this problem, a multiband SHEPWM control technology method for a multilevel inverter based on Walsh functions is proposed. By analyzing multiband SHEPWM for multilevel inverter voltage waveforms under a Fourier series transform, the unified nonlinear multiband SHEPWM equations for a multilevel inverter with a modulation index varying from 0 to 1 can be generalized. The equations can be solved by Walsh function transform. In this way, the difficulties faced in expanding the modulation index, online calculation, and real-time control are resolved simultaneously. A seven-level inverter is taken as the example, for which the piecewise linear equations of Walsh functions under different bands and the trajectories of switching angles are given. Meanwhile, the conditions for multiple sets of solutions at such a point where the modulation index is switched over are also taken into account. The feasibility of the proposed method is verified by simulation based on MATLAB and SIMULINK. Finally, the feasibility of the practical application is proved by the experiment based on Digital Signal Processor (DSP).
\end{abstract}

Keywords: cascaded H-bridge multilevel inverters; walsh functions; multi-band modulation; selected harmonic elimination

\section{Introduction}

The cascaded H-bridge (CHB) multilevel inverter is one of the popular converter topologies used in high-power medium-voltage (MV) drives [1]. It has many advantages, such as its high voltage level, low voltage change rate, and its easy modularization [2]. The pulse width modulation schemes for multilevel inverters can be generally classified into carrier-modulated sine pulse width modulation (SPWM) [3], voltage space vector pulse width modulation (SVPWM) [4], and selected harmonics elimination pulse width modulation (SHEPWM) [5-7]. The SHEPWM scheme has several distinct advantages, such as having a low switching frequency, high output quality, low switching losses, and high inverter efficiency. The core issue of the SHEPWM control is the solution of nonlinear equations of the multilevel inverter, including the selection of initial values and the selection of iterative algorithms. In addition, the traditional trapezoid wave modulation SHEPWM for multilevel inverter control technology is limited by the modulation index, which generally cannot randomly change from 0 to 1 . When the modulation index is relatively low, the SHEPWM nonlinear equations may have no solutions.

At present, most papers use the Newtonian iterative method to solve nonlinear equations, but this needs to find the initial value, which is relatively close to the accurate value, in order to make the algorithm converge, otherwise the iterative algorithm is be convergent. The polynomial synthesis method and the power method are proposed to solve nonlinear equations in $[4,5]$. However. these methods are too complicated and need to be derived for different levels, which makes it hard for them 
to be widely used. The homotopic algorithm is applied to solve the SHEPWM nonlinear equations in [7], which raises the rate of convergence of the iterative process to a certain extent. However, the dependence on the initial value of the iterative process is still strong and there is no guarantee that the iteration can be convergent. A SHEPWM function is proposed based on the Walsh functional transform in [8] by analyzing the relationship between the Walsh domain and the Fourier domain, and by transforming the nonlinear transcendental equations of the Fourier domain into the Walsh function under the domain of the piecewise linear system of equations. The calculation steps of the conversion process are improved and the recursive methods used between the harmonic elimination angles and the fundamental wave voltage section amplitude are proposed in [9-12].

To obtain the initial value, the trial and error method is used in [8]. If there is a lack of evidence when selecting the initial value, the efficiency of the iteration is low. Sometimes equations may also be divergent. The triangular carrier SPWM method is used to get the initial solution in [9]. In fact, this is the method used to solve the reference sine function and a series of piecewise linear functions. However, the calculation process is very troublesome. The approach of Wu is introduced to find the solutions for the initial values of the SHEPWM nonlinear equations in [10], which is complex and hard to achieve.

To expand the modulation index of SHEPWM for a multilevel inverter, the method of reducing a pulse to extend the modulation index to 0.2 is used in [11]. However, the freedom degrees and the number of harmonics eliminated will be reduced at the same time. The polarity reverse method is proposed to expand the modulation index in [12], however the energy consumption control of the DC link needs to be increased to solve the problem of the energy feedback caused by its structure. The six sections of the ladder-type wave switching model are proposed in [13], but the problem of the energy feedback also exists and the working frequency of the switch cannot stay the same.

As it is easy to solve nonlinear equations by using Walsh functions and they have a low dependence on the initial solution, a kind of transformation method is proposed, for which the multilevel inverter modulation index of the multiband SHEPWM modulation scheme [14] can be broadened and combined with Walsh functions. The SHEPWM voltage waveforms of the multilevel inverter in different waveform band modes are analyzed and the multiband SHEPWM solution model with modulation indexes ranging from high to low is established. Finally, the unity SHEPWM equations of the piecewise linear system of equations can be solved using the Walsh function transformation.

This paper is organized as follows: The harmonic elimination model of the multiband SHEPWM is presented in Section 2. Section 3 introduces the Walsh function. The Walsh function transform is proposed to solve the angle of the multiband equation in Section 4. Simulations and experimental results are presented in Sections 5 and 6, respectively, to validate the performance of the proposed method. Finally, the conclusions are summarized in Section 7.

\section{Harmonic Elimination Model for Multiband SHEPWM}

The topology structure of the H-bridge cascaded seven-level inverters is shown in Figure 1. It is composed of a series connection of three $\mathrm{H}$-bridge cells. Each $\mathrm{H}$-bridge unit is powered by a separate DC power supply. Assuming that the input voltages of the three $\mathrm{H}$-bridge cells are $V_{\mathrm{dc} 1}=V_{\mathrm{dc} 2}=V_{\mathrm{dc} 3}$ $=E$, the corresponding output voltages of each cell are $V_{\mathrm{H} 1}, V_{\mathrm{H} 2}, V_{\mathrm{H} 3}$. The generated output voltage of each cell is composed of the three voltage levels $+E,-E$, and 0 , using a different combination of the four switches. 


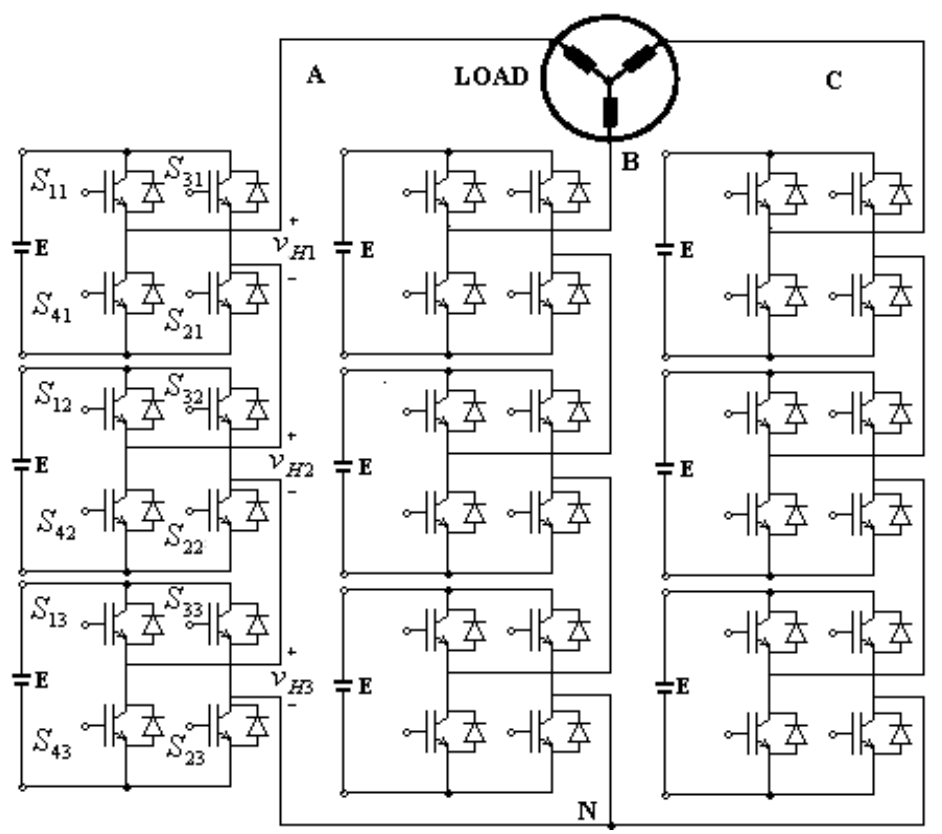

Figure 1. Topology of a cascaded H-bridge (CHB) inverter.

Table 1 shows the output voltage of the inverter under different output functions. Where $S_{1}, S_{2}$ and $\mathrm{S}_{3}$ are the output level function of $\mathrm{H} 1, \mathrm{H} 2$ and $\mathrm{H} 2$ respectively. It can be seen from the table that the phase voltage $V_{\mathrm{AN}}$ of the three-element $\mathrm{CHB}$ inverter canh1 have a total of seven levels of $\pm 3 \mathrm{E}$, $\pm 2 \mathrm{E}, \pm \mathrm{E}$, and 0 . In order to avoid energy circulation, the output states of the opposite polarity of the output voltage of each unit are removed in Table 1. It can be seen from the table that when the output levels of the phase voltage $V_{\mathrm{AN}}$ are $\pm 2 \mathrm{E}$ and $\pm \mathrm{E}$, each unit has a redundant output state function.

Table 1. The outputs corresponding to different output state functions.

\begin{tabular}{|c|c|c|c|c|}
\hline$S_{1} S_{2} S_{3}$ & $V_{\mathrm{H} 1}$ & $V_{\mathrm{H} 2}$ & $V_{\mathrm{H} 3}$ & $V_{\mathrm{AN}}$ \\
\hline 111 & $E$ & $\mathrm{E}$ & $\mathrm{E}$ & $3 E$ \\
\hline 110 & E & E & 0 & \\
\hline 101 & E & 0 & $\mathrm{E}$ & $2 \mathrm{E}$ \\
\hline 011 & 0 & E & $\mathrm{E}$ & \\
\hline 100 & $\mathrm{E}$ & 0 & 0 & \\
\hline 010 & 0 & E & 0 & E \\
\hline 001 & 0 & 0 & E & \\
\hline 000 & 0 & 0 & 0 & 0 \\
\hline-100 & $-E$ & 0 & 0 & \\
\hline $0-10$ & 0 & $-E$ & 0 & $-E$ \\
\hline $00-1$ & 0 & 0 & $-E$ & \\
\hline$-1-10$ & $-E$ & $-E$ & 0 & \\
\hline$-10-1$ & $-E$ & 0 & $-E$ & $-2 \mathrm{E}$ \\
\hline $0-1-1$ & 0 & $-E$ & $-E$ & \\
\hline$-1-1-1$ & $-E$ & $-E$ & $-E$ & $-3 \mathrm{E}$ \\
\hline
\end{tabular}

The multiband SHEPWM multilevel inverter waveform is shown in Figure 2. 


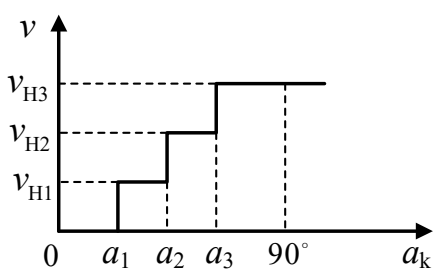

(a)

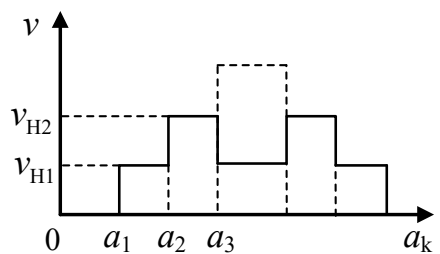

(c)

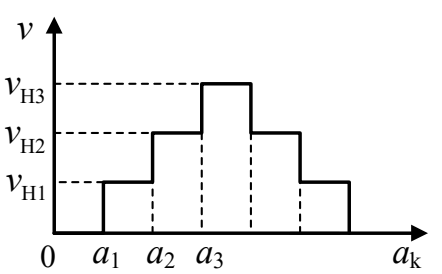

(b)

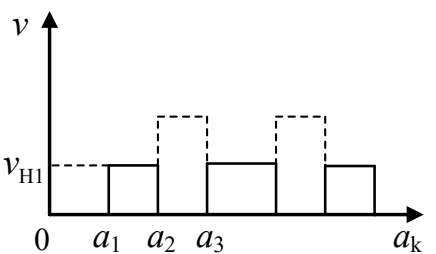

(d)

Figure 2. Multiband SHEPWM waveform for a multilevel inverter: (a) schematic diagram of a seven-level phase voltage; (b) waveform of a seven-level inverter under model A; (c) waveform of a seven-level inverter under model B; (d) waveform of a seven-level inverter under model C.

The graph of the seven-level phase voltage is shown in Figure 2a, which is transformed by a Fourier transform as follows:

$$
u(t)=c_{0}+\sum_{n=1}^{\infty}\left(c_{n} \cos (n \omega t)+b_{n} \sin (n \omega t)\right)
$$

where

$$
\left\{\begin{array}{c}
c_{0}=\frac{1}{T} \int_{0}^{T} u(t) d t \\
c_{n}=\frac{2}{T} \int_{0}^{T} u(t) \cos (n \omega t) d t, n=1,2,3 \cdots
\end{array}\right.
$$

Expanding and analyzing $b n$ in Equation (1) we get:

$$
u(\omega t)=\left\{\begin{array}{c}
b_{n}=0, \quad n \text { is even } \\
b_{n}=\frac{4 E}{n \pi} \sum_{k=1}^{N} p_{k} \cos \left(n \theta_{k}\right), n \text { is odd }
\end{array}\right.
$$

The switching angles denoted by $\theta_{k}$ (in radians) are $0^{0} \leq \theta_{1}<\theta_{2}<\cdots<\theta_{N} \leq 90^{\circ}$. Here, E is the voltage value for each unit of $\mathrm{dc} ; b_{n}$ is the harmonic coefficient; $p_{k}$ is marked +1 when it rises at the point of $\theta_{k}$ and -1 when it decline. The graph of the half-wave symmetry waveform of the seven-level ladder-type modulation is shown in Figure 2b, in which the output voltages of each unit H-bridge are $V_{\mathrm{H} 1}, V_{\mathrm{H} 2}$, and $V_{\mathrm{H} 3}$. According to Equation (3), a harmonic elimination of nonlinear equations can be established. There are no solutions when the modulation index is lower than 0.4 , as the five- and seven-fold harmonics are eliminated by the numerical algorithm. According to the above situation, a multiband modulation scheme is proposed in this paper by broadening the index of modulation. The modulation index can be broadened as shown in Figure 2c, d.

By observing the staircase waveform, it is easy to find that the waveform transformation rules between different wavebands are used to fold down the waveform with the dotted line at the top. The applicable modulation indexes shown in Figure $2 \mathrm{~b}$ to Figure $2 \mathrm{~d}$ for modulation modes $\mathrm{A}, \mathrm{B}$, and $\mathrm{C}$ are [0.4, 1], [0.3, 0.3], and [0, 0.3], respectively. The modulation index can be broadened to 0-1 according to the above multiband modulation method. The correct switch angles under different modulation indexes can be obtained by establishing SHEPWM nonlinear equations in accordance with the waveforms of different wavebands, for which the modulation index has different ranges. 


\section{Walsh Functions}

The Walsh function is a standard orthogonal function system. It has only two values, 0 and 1 , but the value of the function changes between 0 and 1 , so the theoretical analysis is more complex than the trigonometric function. In recent years, due to the rapid development of new disciplines and technologies, Walsh function theory has been widely used in radar, communication, medicine, imaging, and other fields. The introduction of the Walsh function into the electrical field is of great significance.

The Walsh functions are a set of complete orthogonal function systems that can be expressed as Wal $(n, t)$, where $\mathrm{n}$ is usually the zero crossing point $(\mathrm{ZCP})$ number under time $\mathrm{T}$ of a certain order for the definition of Walsh functions, where $t$ is the normalized time spacing of $\mathrm{T}$.

The quarter-cycle waveform normalized by Figure 2a is shown in Figure 3, where $T(0-1)$ is divided into $2^{\mathrm{m}}$ uniform areas, $\mathrm{c}$ is the normalized value of $\theta_{1}$, and the interval number of the rising edge is marked with N. Similar to Fourier expressions, the Walsh functions are obtained using the waveform transformed by the Walsh function, which are shown below:

$$
\begin{aligned}
& f(t)=a_{0}+W_{\mathrm{a} 1}(0, t)+\sum_{i=1}^{N-1} a_{i} W_{\mathrm{a} 1}(i, t) \\
& \left\{\begin{array}{c}
a_{0}=\frac{2}{T} \int_{0}^{T} f(t) W_{\mathrm{a} 1}(0, t) d t, i=1,2,3, \cdots \\
a_{1}=\frac{1}{T} \int_{0}^{T} f(t) W_{\mathrm{a} 1}(i, t) d t, i=1,2,3, \cdots
\end{array}\right. \\
& \begin{array}{r}
1 \\
0
\end{array}
\end{aligned}
$$

Figure 3. A quarter cycle of a normalized three-level Pulse width modulation (PWM) waveform.

Equation (4) is simplified according to the symmetry as follows:

$$
f(t)=\sum_{i=1}^{N / 2} W_{4 i-3} W_{\mathrm{a} 1}(4 i-3, t)
$$

In Equation (6), $W_{4 i-3}=F^{w}(4 i-3)$ is the coefficient of $W_{\mathrm{a} 1}(4 i-3, t)$, meaning that the corresponding waveform of the Walsh functions is $f(t)$. A quarter cycle of the normalized three-level PWM waveform is shown in Figure 2. According to Figure 2, Equation (7) can be deduced as follows:

$$
\begin{gathered}
F^{w}(4 i-3) \quad=\int_{0}^{1} f(t) W_{\mathrm{a} 1}(4 i-3, t) d t \\
=\frac{1}{2^{m-2}} \sum_{n=N+1}^{2^{m} / 4} W_{\mathrm{a} 1}(4 i-3, n)+\left(\frac{N}{2^{m-2}}-4 c\right) W_{\mathrm{a} 1}(4 i-3, N), i=1,2,3, \cdots
\end{gathered}
$$

As seen in Equations (1) and (4), some transformations can be taken to convert the transcendental equations under the domain of the Fourier transform into piecewise linear equations under the domain of the Walsh function. According to the Equation (3), we can get:

$$
\begin{gathered}
u(w t)=f(w t)=\sum_{n=1}^{\infty} b_{2 n-1} \sin [(2 n-1) w t] \\
b_{2 n-1}=\frac{8}{\pi} \int_{0}^{\pi / 4} f(w t) \sin [(2 n-1) w t] d(w t), n=1,2,3, \cdots
\end{gathered}
$$


Under the same angular frequency, Equation (6) can be developed under the domain of the Walsh function to infinity, Equation (8) can be made equivalent with it under the Fourier transform, then Equation (6) can be brought into Equation (9), and we can get:

$$
b_{2 n-1}=\frac{8}{\pi} \int_{0}^{\pi / 4} \sum_{i=1}^{\infty} W_{4 i-3} W_{\mathrm{a} 1}(4 i-3, w t) \sin [(2 n-1) w t] d(w t), n=0,1,2,3, \cdots
$$

Assuming that:

$$
B_{2 n-1}\left(W_{\mathrm{a} 1}(4 i-3, w t)\right)=\frac{8}{\pi} \int_{0}^{\pi / 4} W_{\mathrm{a} 1}(4 i-3, w t) \sin [(2 n-1) w t] d(w t)
$$

Equation (11) is the Fourier coefficient of the $(2 n-1)$ harmonic for the Walsh function $W_{\mathrm{a} 1}(4 i-3, w t)$, then we plug in Equation (10) into the deformation:

$$
b_{2 n-1}=\sum_{i=1}^{\infty} W_{4 i-3} B_{2 n-1}\left[W_{\mathrm{a} 1}(4 i-3, w t)\right]
$$

Equation (11) is complex to solve, while a nonrecursive formula of the Walsh function Fourier transformation is given in $[15,16]$. For $k=2 i-1, i=1,2,3, \ldots, m=4 n-3, n=1,2,3, \ldots$ :

$$
B_{k}\left(W_{\mathrm{a} 1}(m, w t)\right)=2(-1)^{g_{0}}(-j)^{d} \times\left[\prod_{x=0}^{M-1} \cos \left(\frac{\pi k}{2^{x+1}}-\frac{\pi g_{x}}{2}\right)\right] \frac{\sin \left(\pi k / 2^{M}\right)}{\pi k / 2^{M}}
$$

In which $j=(-1)^{1 / 2}$ records only plus or minus of $(-j)^{d}$ when calculating, while the variable instructions of Equation (13) are as follows:

1. Record the order of the Walsh function as $m$, making its expressions for e binary;

2. Convert e as gray code $g$, the record the $x$ bite of the gray code for $g_{x}$;

3. $\mathrm{M}$ is the total number of $g$, while $d$ is the total number of 1 contained in $g$.

\section{Solving Equations}

Summarizing Equation (11):

$$
[\mathrm{b}]=[\mathrm{U}]=[\mathrm{B}][\mathrm{F}]
$$

In this process, [b] is the harmonic coefficient matrix, which is replaced by [U] for simplification. The matrixes $[\mathrm{B}]$ and $[\mathrm{F}]$ are the Walsh transform matrix and Walsh coefficient matrix.

We assume that the harmonic coefficient $U_{k}$ of the $\mathrm{k}$ time eliminated harmonic component is zero, which satisfies the fundamental current amplitude at the same time. We then establish a harmonic elimination system for linear equations and solve the switch angle, the calculation steps for which are shown as follows:

1. Determine the total interval number $\mathrm{N}$ according to the precision of the harmonic elimination, give a set of relatively accurate initial values, determine the interval after normalization, and serve the initial conditions;

2. According to interval number $\mathrm{N}$ and the harmonic number $\mathrm{k}$, calculate the Walsh transform matrix $[\mathrm{B}]$

3. According to interval number $\mathrm{N}$ and the determined initial conditions, calculate the Walsh coefficient matrix [F];

4. According to Equation (14), establish the piecewise linear system of equations;

5. Solve the piecewise linear system of equations and get the corresponding number of switch angles. 
Taking the seven-level waveband $\mathrm{A}$ as an example, the accuracy of the interval number $\mathrm{N}$ is shown as 64 [16], the initial switch angles for $a_{1}, a_{2}$, and $a_{3}$ are $11.52^{\circ}, 28.728^{\circ}$, and $57.096^{\circ}$. The interval numbers of the switch angles are 3,6 , and 11 , respectively. The scope of the initial angles is shown as:

$$
2 \times 360 / 64 \leq a_{1} \leq 3 \times 360 / 64,5 \times 360 / 64 \leq a_{2} \leq 6 \times 360 / 64,10 \times 360 / 64 \leq a_{3} \leq 11 \times 360 / 64
$$

For the seven-level inverter's three-phase system, five and seven-fold harmonics are generally eliminated. The Walsh coefficient matrix [F] can be obtained by the selection of the initial conditions and by using Equation (7), as shown in Table 2. The corresponding coefficient of the Walsh function is $F^{w}(4 i-3)$, where $i=1,2,3 \ldots$, and using the interval number $\mathrm{N}$ and the eliminated harmonic number $\mathrm{k}$, the Walsh transform matrix [B] can be obtained, as shown in Table 3. As space is limited, only some of the data are shown.

Table 2. Walsh coefficient matrix [F].

\begin{tabular}{|c|c|c|c|}
\hline$F^{\mathrm{W}}(1)$ & $-4 a_{1}-4 a_{2}-4 a_{3}+3$ & $F^{\mathrm{W}}(5)$ & $-4 a_{1}-4 a_{2}+4 a_{3}-1$ \\
\hline$F^{\mathrm{w}}(9)$ & $-4 a_{1}+4 a_{2}+4 a_{3}-1$ & $F^{\mathrm{W}}(13)$ & $-4 a_{1}+4 a_{2}-4 a_{3}$ \\
\hline$F^{\mathrm{w}}(17)$ & $4 a_{1}+4 a_{2}+4 a_{3}-5 / 4$ & $F^{\mathrm{W}}(21)$ & $4 a_{1}+4 a_{2}-4 a_{3}+1 / 4$ \\
\hline$F^{\mathrm{w}}(25)$ & $4 a_{1}-4 a_{2}-4 a_{3}+3 / 4$ & $F^{\mathrm{w}}(29)$ & $4 a_{1}-4 a_{2}+4 a_{3}-3 / 4$ \\
\hline$F^{\mathrm{w}}(33)$ & $4 a_{1}+4 a_{2}+4 a_{2}-9 / 8$ & $F^{\mathrm{w}}(37)$ & $4 a_{1}+4 a_{2}-4 a_{3}+1 / 8$ \\
\hline$F^{\mathrm{w}}(41)$ & $4 a_{1}-4 a_{2}-4 a_{3}+7 / 8$ & $F^{\mathrm{w}}(45)$ & $4 a_{1}-4 a_{2}+4 a_{3}-3 / 8$ \\
\hline$F^{\mathrm{w}}(49)$ & $-4 a_{1}-4 a_{2}-4 a_{3}+9 / 8$ & $F^{\mathrm{w}}(53)$ & $-4 a_{1}-4 a_{2}+4 a_{3}-1 / 8$ \\
\hline$F^{\mathrm{w}}(57)$ & $-4 a_{1}+4 a_{2}+4 a_{3}-7 / 8$ & $F^{\mathrm{w}}(61)$ & $-4 a_{1}+4 a_{2}-4 a_{3}+3 / 8$ \\
\hline
\end{tabular}

Table 3. Walsh coefficient matrix [B].

\begin{tabular}{|c|c|c|c|c|c|c|c|c|c|c|c|c|c|c|c|c|}
\hline$k$ & $W_{\mathrm{al}}(1, t)$ & $W_{\mathrm{al}}(5, t)$ & $W_{\mathrm{al}}(9, t)$ & $W_{\mathrm{al}}(13, t)$ & $W_{\mathrm{al}}(17, t)$ & $W_{\mathrm{al}}(21, t)$ & $W_{\mathrm{al}}(25, t)$ & $W_{\mathrm{al}}(29, t)$ & $W_{\mathrm{al}}(33, t)$ & $W_{\mathrm{al}}(37, t)$ & $W_{\mathrm{al}}(41, t)$ & $W_{\mathrm{al}}(45, t)$ & $W_{\mathrm{al}}(49, t)$ & $W_{\mathrm{al}}(53, t)$ & $W_{\mathrm{al}}(57, t)$ & $W_{\mathrm{al}}(61, t)$ \\
\hline 1 & 1.2732 & -0.5274 & -0.1049 & -0.2533 & -0.0249 & 0.0103 & -0.0519 & -0.1254 & -0.0062 & 0.0026 & 0.0005 & 0.0012 & -0.0124 & 0.0052 & -0.0259 & -0.0626 \\
\hline 7 & 0.1819 & -0.0753 & 0.3788 & 0.9144 & -0.7505 & 0.3108 & 0.0618 & 0.1493 & -0.0534 & 0.0221 & -0.1112 & -0.2685 & -0.3272 & 0.1355 & 0.0270 & 0.0651 \\
\hline 11 & 0.1157 & 0.2794 & 0.4182 & -0.1732 & 0.3241 & 0.7824 & -0.5228 & 0.2166 & -0.1298 & -0.3134 & -0.4690 & 0.1943 & 0.1038 & 0.2507 & -0.1675 & 0.0694 \\
\hline
\end{tabular}

Combined with Equation (14):

$$
\begin{gathered}
U_{1}=4.5327-1.9431 a_{1}-4.1112 a_{2}-6.8591 a_{3}, U_{5}=-0.6268-7.4570 a_{1}-3.3862 a_{2}+7.1595 a_{3}, \\
U_{7}=0.8745-7.7586 a_{1}+4.6724 a_{2}-6.3000 a_{3}
\end{gathered}
$$

Assuming that the harmonic coefficients $\mathrm{U}_{5}$ and $\mathrm{U}_{7}$ are zero, according to Equation (16):

$$
a_{1}=-0.0140 U_{1}+0.0748, a_{2}=-0.1186 U_{1}+0.4422, a_{3}=-0.0707 U_{1}+0.3746
$$

According to Equations (15) and (17), we get $1.4172 \leq U_{1} \leq 1.4523$, which meets the fundamental current amplitude conditions. Thus, the first set of solutions can be obtained. Then, the limit value, which met Equation (17), should be substituted into Equation (16), and the other set of switch angles can be obtained. After determining the range, the process above can be repeated and another set of solutions can be obtained. All switch angles can also be obtained.

Taking the seven-level inverter multiband ladder-type modulation as an example, the switching angles for SHEPWM can be solved using Walsh transforms. The selected interval number $\mathrm{N}$ is 64 and the eliminated 5th and 7th harmonic are calculated in accordance with the above example. Along with the multiband modulation mode, as shown in Figure 2, the harmonic elimination switch angles of A, B, and C mode can be obtained, as shown in Tables 4-6. 
Table 4. Results of A waveband switching angles.

\begin{tabular}{|c|c|c|c|}
\hline $\begin{array}{l}\text { Range of Fundamental } \\
\text { Amplitude }\left(U_{1}\right)\end{array}$ & $\begin{array}{c}\text { Switch Angels }\left(a_{1}, a_{2}, a_{3}\right) \\
\text { after Normalization }\end{array}$ & $\begin{array}{c}\text { Range of Fundamental } \\
\text { Amplitude }\left(U_{1}\right)\end{array}$ & $\begin{array}{c}\text { Switch Angels }\left(a_{1}, a_{2}, a_{3}\right) \\
\text { after Normalization }\end{array}$ \\
\hline $3.2202-3.1742$ & $\begin{array}{c}a_{1}=0.2442 U_{1}-0.7395 \\
a_{2}=-0.3261 U_{1}+1.0976 \\
a_{3}=-0.0928 U_{1}+0.4430\end{array}$ & $2.4257-2.2879$ & $\begin{array}{l}a_{1}=-0.1134 U_{1}+0.3532 \\
a_{2}=-0.0096 U_{1}+0.1747 \\
a_{3}=-0.0653 U_{1}+0.3352\end{array}$ \\
\hline $3.1738-3.0868$ & $\begin{array}{c}a_{1}=0.0288 U_{1}-0.0557 \\
a_{2}=-0.1433 U_{1}+0.5173 \\
a_{3}=-0.0881 U_{1}+0.4282\end{array}$ & $2.2870-2.2762$ & $\begin{array}{c}a_{1}=-0.1197 U_{1}+0.3675 \\
a_{2}=0.0880 U_{1}-0.0484 \\
a_{3}=-0.1376 U_{1}+0.5007\end{array}$ \\
\hline $3.0839-3.0736$ & $\begin{array}{c}a_{1}=0.0731 U_{1}-0.1933 \\
a_{2}=-0.1749 U_{1}+0.6157 \\
a_{3}=-0.0793 U_{1}+0.4008\end{array}$ & $2.2769-2.1240$ & $\begin{array}{c}a_{1}=-0.0734 U_{1}+0.2623 \\
a_{2}=0.0186 U_{1}+0.1093 \\
a_{3}=-0.1022 U_{1}+0.4202\end{array}$ \\
\hline $3.0698-2.9380$ & $\begin{array}{l}a_{1}=-0.0140 U_{1}+0.0748 \\
a_{2}=-0.1186 U_{1}+0.4422 \\
a_{3}=-0.0707 U_{1}+0.3746\end{array}$ & $2.1236-1.9870$ & $\begin{array}{l}a_{1}=-0.0212 U_{1}+0.1515 \\
a_{2}=-0.0297 U_{1}+0.2120 \\
a_{3}=-0.0912 U_{1}+0.3968\end{array}$ \\
\hline $2.9363-2.8491$ & $\begin{array}{l}a_{1}=-0.0588 U_{1}+0.2064 \\
a_{2}=-0.1060 U_{1}+0.4050 \\
a_{3}=-0.0555 U_{1}+0.3300\end{array}$ & $1.9825-1.9538$ & $\begin{array}{l}a_{1}=-0.0214 U_{1}+0.1518 \\
a_{2}=-0.0215 U_{1}+0.1956 \\
a_{3}=-0.0963 U_{1}+0.4069\end{array}$ \\
\hline $2.8485-2.7871$ & $\begin{array}{l}a_{1}=-0.0425 U_{1}+0.1599 \\
a_{2}=-0.1037 U_{1}+0.3984 \\
a_{3}=-0.0586 U_{1}+0.3388\end{array}$ & $1.9526-1.9324$ & $\begin{array}{c}a_{1}=0.0270 U_{1}+0.0572 \\
a_{2}=-0.0757 U_{1}+0.3015 \\
a_{3}=-0.0833 U_{1}+0.3814\end{array}$ \\
\hline $2.7887-2.7219$ & $\begin{array}{l}a_{1}=-0.0826 U_{1}+0.2717 \\
a_{2}=-0.1201 U_{1}+0.4443 \\
a_{3}=-0.0269 U_{1}+0.2504\end{array}$ & $1.9270-1.9169$ & $\begin{array}{c}a_{1}=0.0305 U_{1}+0.0506 \\
a_{2}=-0.0680 U_{1}+0.2866 \\
a_{3}=-0.0898 U_{1}+0.3936\end{array}$ \\
\hline $2.7212-2.6417$ & $\begin{array}{l}a_{1}=-0.0738 U_{1}+0.2477 \\
a_{2}=-0.0974 U_{1}+0.3823 \\
a_{3}=-0.0385 U_{1}+0.2820\end{array}$ & $1.9172-1.8738$ & $\begin{array}{l}a_{1}=-0.0101 U_{1}+0.1283 \\
a_{2}=-0.0574 U_{1}+0.2663 \\
a_{3}=-0.0706 U_{1}+0.3572\end{array}$ \\
\hline $2.6424-2.5541$ & $\begin{array}{c}a_{1}=-0.1099 U_{1}+0.3432 \\
a_{2}=-0.1373 U_{1}+0.4878 \\
a_{3}=0.0151 U_{1}+0.1402\end{array}$ & $1.8994-1.7432$ & $\begin{array}{l}a_{1}=-0.0077 U_{1}+0.1240 \\
a_{2}=-0.0555 U_{1}+0.2627 \\
a_{3}=-0.0731 U_{1}+0.3618\end{array}$ \\
\hline $2.5545-2.4011$ & $\begin{array}{c}a_{1}=-0.1019 U_{1}+0.3228 \\
a_{2}=-0.0762 U_{1}+0.3320 \\
a_{3}=-0.0276 U_{1}+0.2494 \\
a_{1}=-0.1314 U_{1}+0.3968 \\
a_{2}=-0.1281 U_{1}+0.4621 \\
a_{3}=0.0376 U_{1}+0.0856\end{array}$ & $1.7439-1.5053$ & $\begin{array}{c}a_{1}=0.0038 U_{1}+0.1038 \\
a_{2}=-0.0725 U_{1}+0.2924 \\
a_{3}=-0.0655 U_{1}+0.3486 \\
- \\
- \\
-\end{array}$ \\
\hline
\end{tabular}

Table 5. Results of B waveband switching angles.

\begin{tabular}{cc}
\hline Range of Fundamental Amplitude $\left(U_{1}\right)$ & Switch Angels $\left(a_{1}, a_{2}, a_{3}\right)$ after Normalization \\
\hline $1.4410-1.4066$ & $a_{1}=-0.0201 U_{1}+0.1430, a_{2}=-0.0669 U_{1}+0.2816, a_{3}=0.0517 U_{1}+0.1755$ \\
$1.4288-1.2282$ & $a_{1}=-0.0332 U_{1}+0.1623, a_{2}=-0.0779 U_{1}+0.2988, a_{3}=0.0294 U_{1}+0.2062$ \\
$1.2268-1.1439$ & $a_{1}=-0.0410 U_{1}+0.1719, a_{2}=-0.1216 U_{1}+0.3523, a_{3}=-0.0205 U_{1}+0.2674$ \\
$1.1417-1.0902$ & $a_{1}=-0.0381 U_{1}+0.1685, a_{2}=-0.1014 U_{1}+0.3293, a_{3}=-0.0015 U_{1}+0.2458$ \\
$1.0904-1.0538$ & $a_{1}=-0.0415 U_{1}+0.1722, a_{2}=-0.2549 U_{1}+0.4967, a_{3}=-0.1580 U_{1}+0.4165$ \\
\hline
\end{tabular}

Table 6. Results of $\mathrm{C}$ waveband switching angles when $\mathrm{N}=64$.

\begin{tabular}{cc}
\hline Range of Fundamental Amplitude $\left(\boldsymbol{U}_{\mathbf{1}}\right)$ & Switch Angels $\left(\boldsymbol{a}_{\mathbf{1}}, \boldsymbol{a}_{\mathbf{2}}, \boldsymbol{a}_{\mathbf{3}}\right)$ after Normalization \\
\hline $1.1416-1.0473$ & $a_{1}=0.0384 U_{1}-0.0022, a_{2}=0.1656 U_{1}+0.0297, a_{3}=0.0265 U_{1}+0.1995$ \\
$1.0481-0.9226$ & $a_{1}=0.0318 U_{1}+0.0047, a_{2}=0.0816 U_{1}+0.1176, a_{3}=-0.0565 U_{1}+0.2865$ \\
$0.9219-0.8648$ & $a_{1}=0.0298 U_{1}+0.0065, a_{2}=0.0947 U_{1}+0.1056, a_{3}=-0.0432 U_{1}+0.2742$ \\
$0.8658-0.8030$ & $a_{1}=0.0165 U_{1}+0.0180, a_{2}=0.0745 U_{1}+0.1230, a_{3}=-0.0618 U_{1}+0.2902$ \\
$0.8062-0.6579$ & $a_{1}=0.0209 U_{1}+0.0144, a_{2}=0.0749 U_{1}+0.1226, a_{3}=-0.0605 U_{1}+0.2893$ \\
$0.6560-0.6484$ & $a_{1}=-0.0035 U_{1}+0.0304, a_{2}=0.0710 U_{1}+0.1253, a_{3}=-0.0637 U_{1}+0.2913$ \\
\hline
\end{tabular}


The linear equations transformed by Walsh functions cannot be solved when the seven-level inverter modulation index of 64 under the $C$ band mode is below 0.170 , as shown in Table 5 . To solve this problem, the solution involving expanding the interval number is adopted, so that the interval number $\mathrm{N}$ is taken as 128 and the $\mathrm{C}$ band mode switch angles can be evaluated, as shown in Table 7 .

Table 7. Results of $\mathrm{C}$ waveband switching angles when $\mathrm{N}=128$.

\begin{tabular}{|c|c|c|c|}
\hline $\begin{array}{l}\text { Range of Fundamental } \\
\text { Amplitude }\left(U_{1}\right)\end{array}$ & Switch Angels $\left(a_{1}, a_{2}, a_{3}\right)$ after Normalization & $\begin{array}{l}\text { Range of Fundamental } \\
\text { Amplitude }\left(U_{1}\right)\end{array}$ & $\begin{array}{c}\text { Switch Angels }\left(a_{1}, a_{2}, a_{3}\right) \\
\text { after Normalization }\end{array}$ \\
\hline $1.1869-1.1867$ & $\begin{array}{c}a_{1}=-41.3455 U_{1}+49.0735 \\
a_{2}=-7.4786 U_{1}+8.9118 \\
a_{3}=-1.8609 U_{1}+2.2777\end{array}$ & $0.8708-0.8319$ & $\begin{array}{l}a_{1}=-0.2008 U_{1}+0.2608 \\
a_{2}=-0.1062 U_{1}+0.2033 \\
a_{3}=-0.1063 U_{1}+0.2415\end{array}$ \\
\hline $1.1867-1.1865$ & $\begin{array}{c}a_{1}=-15.2520 U_{1}+18.1081 \\
a_{2}=-7.9191 U_{1}+9.4347 \\
a_{3}=-1.8210 U_{1}+2.2305\end{array}$ & $0.8319-0.8234$ & $\begin{array}{l}a_{1}=-0.3251 U_{1}+0.3642 \\
a_{2}=-0.2547 U_{1}+0.3269 \\
a_{3}=-0.1275 U_{1}+0.2591\end{array}$ \\
\hline $1.1864-1.1862$ & $\begin{array}{c}a_{1}=-9.4425 U_{1}+11.2151 \\
a_{2}=-5.3968 U_{1}+6.4420 \\
a_{3}=-2.1462 U_{1}+2.6162\end{array}$ & $0.8234-0.8094$ & $\begin{array}{l}a_{1}=-0.2590 U_{1}+0.3098 \\
a_{2}=-0.2145 U_{1}+0.2938 \\
a_{3}=-0.1514 U_{1}+0.2788\end{array}$ \\
\hline $1.1862-1.1860$ & $\begin{array}{l}a_{1}=-6.7262 U_{1}+7.9930 \\
a_{2}=-3.3420 U_{1}+4.0046 \\
a_{3}=-1.1600 U_{1}+1.4463\end{array}$ & $0.8090-0.8033$ & $\begin{array}{l}a_{1}=-0.2296 U_{1}+0.2860 \\
a_{2}=-0.1563 U_{1}+0.2467 \\
a_{3}=-0.1191 U_{1}+0.2526\end{array}$ \\
\hline $1.1860-1.1845$ & $\begin{array}{l}a_{1}=-5.0229 U_{1}+5.9730 \\
a_{2}=-3.8515 U_{1}+4.6089 \\
a_{3}=-1.1952 U_{1}+1.4882\end{array}$ & $0.8033-0.7915$ & $\begin{array}{l}a_{1}=-0.3709 U_{1}+0.3995 \\
a_{2}=-0.3290 U_{1}+0.3854 \\
a_{3}=-0.1464 U_{1}+0.2745\end{array}$ \\
\hline $1.1844-1.1822$ & $\begin{array}{l}a_{1}=-3.0451 U_{1}+3.6302 \\
a_{2}=-3.3748 U_{1}+4.0443 \\
a_{3}=-1.4747 U_{1}+1.8192\end{array}$ & $0.7794-0.7616$ & $\begin{array}{l}a_{1}=-0.2836 U_{1}+0.3304 \\
a_{2}=-0.2769 U_{1}+0.3442 \\
a_{3}=-0.1789 U_{1}+0.3003\end{array}$ \\
\hline $1.1821-1.1818$ & $\begin{array}{l}a_{1}=-1.9737 U_{1}+2.3637 \\
a_{2}=-3.0006 U_{1}+3.6017 \\
a_{3}=-1.9294 U_{1}+2.3566\end{array}$ & $0.7795-0.7706$ & $\begin{array}{l}a_{1}=-0.4671 U_{1}+0.4735 \\
a_{2}=-0.5118 U_{1}+0.5272 \\
a_{3}=-0.2255 U_{1}+0.3366\end{array}$ \\
\hline $1.1818-1.1811$ & $\begin{array}{l}a_{1}=-2.1677 U_{1}+2.5930 \\
a_{2}=-3.5741 U_{1}+4.2796 \\
a_{3}=-2.0826 U_{1}+2.5378\end{array}$ & $0.7707-0.7666$ & $\begin{array}{l}a_{1}=-0.3392 U_{1}+0.3749 \\
a_{2}=-0.4585 U_{1}+0.4862 \\
a_{3}=-0.2967 U_{1}+0.3915\end{array}$ \\
\hline $1.1810-1.1789$ & $\begin{array}{l}a_{1}=-1.4996 U_{1}+1.8038 \\
a_{2}=-1.9516 U_{1}+2.3632 \\
a_{3}=-1.0120 U_{1}+1.2733\end{array}$ & $0.7666-0.7582$ & $\begin{array}{l}a_{1}=-0.2816 U_{1}+0.3307 \\
a_{2}=-0.3179 U_{1}+0.3784 \\
a_{3}=-0.2089 U_{1}+0.3242\end{array}$ \\
\hline $1.1790-1.1757$ & $\begin{array}{l}a_{1}=-0.9903 U_{1}+1.2034 \\
a_{2}=-1.8240 U_{1}+2.2130 \\
a_{3}=-1.3131 U_{1}+1.6283\end{array}$ & $0.7582-0.7522$ & $\begin{array}{l}a_{1}=-0.4541 U_{1}+0.4615 \\
a_{2}=-0.5405 U_{1}+0.5472 \\
a_{3}=-0.2542 U_{1}+0.3586\end{array}$ \\
\hline $1.1756-1.1748$ & $\begin{array}{l}a_{1}=-1.1810 U_{1}+1.4275 \\
a_{2}=-2.1990 U_{1}+2.6537 \\
a_{3}=-1.4225 U_{1}+1.7569\end{array}$ & $0.7521-0.7385$ & $\begin{array}{l}a_{1}=-0.3132 U_{1}+0.3555 \\
a_{2}=-0.4749 U_{1}+0.4978 \\
a_{3}=-0.3257 U_{1}+0.4124\end{array}$ \\
\hline $1.1749-1.1748$ & $\begin{array}{l}a_{1}=-0.7821 U_{1}+0.9590 \\
a_{2}=-2.8460 U_{1}+3.4141 \\
a_{3}=-2.4265 U_{1}+2.9366\end{array}$ & $0.7383-0.7357$ & $\begin{array}{l}a_{1}=-0.2448 U_{1}+0.3051 \\
a_{2}=-0.3101 U_{1}+0.3761 \\
a_{3}=-0.2246 U_{1}+0.3377\end{array}$ \\
\hline $1.1747-1.1681$ & $\begin{array}{l}a_{1}=-0.5533 U_{1}+0.6901 \\
a_{2}=-1.1432 U_{1}+1.4135 \\
a_{3}=-0.9186 U_{1}+1.1650\end{array}$ & $0.7356-0.7350$ & $\begin{array}{l}a_{1}=-0.3861 U_{1}+0.4090 \\
a_{2}=-0.4911 U_{1}+0.5094 \\
a_{3}=-0.2604 U_{1}+0.3640\end{array}$ \\
\hline $1.1682-1.1670$ & $\begin{array}{l}a_{1}=-0.3997 U_{1}+0.5107 \\
a_{2}=-1.6291 U_{1}+1.9812 \\
a_{3}=-1.5353 U_{1}+1.8855\end{array}$ & $0.7350-0.7165$ & $\begin{array}{l}a_{1}=-0.2548 U_{1}+0.3126 \\
a_{2}=-0.4231 U_{1}+0.4594 \\
a_{3}=-0.3206 U_{1}+0.4083\end{array}$ \\
\hline $1.1671-1.1586$ & $\begin{array}{l}a_{1}=-0.2959 U_{1}+0.3897 \\
a_{2}=-0.6242 U_{1}+0.8085 \\
a_{3}=-0.6143 U_{1}+0.8107\end{array}$ & $0.7163-0.7136$ & $\begin{array}{l}a_{1}=-0.1849 U_{1}+0.2625 \\
a_{2}=-0.4672 U_{1}+0.4909 \\
a_{3}=-0.4319 U_{1}+0.4879\end{array}$ \\
\hline $1.1581-1.1577$ & $\begin{array}{l}a_{1}=-0.3442 U_{1}+0.4455 \\
a_{2}=-0.7335 U_{1}+0.9351 \\
a_{3}=-0.6575 U_{1}+0.8607\end{array}$ & $0.7139-0.6966$ & $\begin{array}{l}a_{1}=-0.1378 U_{1}+0.2288 \\
a_{2}=-0.2474 U_{1}+0.3341 \\
a_{3}=-0.2562 U_{1}+0.3626\end{array}$ \\
\hline $1.1575-1.1558$ & $\begin{array}{l}a_{1}=-0.2594 U_{1}+0.3473 \\
a_{2}=-1.0795 U_{1}+1.3355 \\
a_{3}=-1.0788 U_{1}+1.3484\end{array}$ & $0.6967-0.6895$ & $\begin{array}{l}a_{1}=-0.1893 U_{1}+0.2647 \\
a_{2}=-0.3303 U_{1}+0.3918 \\
a_{3}=-0.2852 U_{1}+0.3828\end{array}$ \\
\hline $1.1558-1.1411$ & $\begin{array}{l}a_{1}=-0.1997 U_{1}+0.2785 \\
a_{2}=-0.3944 U_{1}+0.5438 \\
a_{3}=-0.4443 U_{1}+0.6151\end{array}$ & $0.6896-0.6860$ & $\begin{array}{l}a_{1}=-0.1361 U_{1}+0.2280 \\
a_{2}=-0.3626 U_{1}+0.4141 \\
a_{3}=-0.3691 U_{1}+0.4407\end{array}$ \\
\hline
\end{tabular}


Table 7. Cont.

\begin{tabular}{|c|c|c|c|}
\hline $\begin{array}{l}\text { Range of Fundamental } \\
\text { Amplitude }\left(U_{1}\right)\end{array}$ & Switch Angels $\left(a_{1}, a_{2}, a_{3}\right)$ after Normalization & $\begin{array}{l}\text { Range of Fundamental } \\
\text { Amplitude }\left(U_{1}\right)\end{array}$ & $\begin{array}{l}\text { Switch Angels }\left(a_{1}, a_{2}, a_{3}\right) \\
\text { after Normalization }\end{array}$ \\
\hline 1.1411-1. 1393 & $\begin{array}{l}a_{1}=-0.1654 U_{1}+0.2393 \\
a_{2}=-0.6211 U_{1}+0.8025 \\
a_{3}=-0.7001 U_{1}+0.9070\end{array}$ & $0.6858-0.6499$ & $\begin{array}{l}a_{1}=-0.0994 U_{1}+0.2028 \\
a_{2}=-0.1763 U_{1}+0.2863 \\
a_{3}=-0.2177 U_{1}+0.3368\end{array}$ \\
\hline $1.1392-1.1133$ & $\begin{array}{l}a_{1}=-0.1371 U_{1}+0.2070 \\
a_{2}=-0.2000 U_{1}+0.3228 \\
a_{3}=-0.3019 U_{1}+0.4533\end{array}$ & $0.6501-0.6486$ & $\begin{array}{l}a_{1}=-0.0714 U_{1}+0.1847 \\
a_{2}=-0.0816 U_{1}+0.2248 \\
a_{3}=-0.1495 U_{1}+0.2925\end{array}$ \\
\hline $1.1133-1.1101$ & $\begin{array}{l}a_{1}=-0.1155 U_{1}+0.1829 \\
a_{2}=-0.0616 U_{1}+0.1687 \\
a_{3}=-0.1802 U_{1}+0.3178\end{array}$ & $0.6487-0.6109$ & $\begin{array}{l}a_{1}=-0.0602 U_{1}+0.1774 \\
a_{2}=-0.0856 U_{1}+0.2274 \\
a_{3}=-0.1642 U_{1}+0.3020\end{array}$ \\
\hline $1.1109-1.0968$ & $\begin{array}{l}a_{1}=-0.1309 U_{1}+0.2001 \\
a_{2}=-0.0965 U_{1}+0.2074 \\
a_{3}=-0.1935 U_{1}+0.3326\end{array}$ & $0.6112-0.6028$ & $\begin{array}{l}a_{1}=-0.0762 U_{1}+0.1872 \\
a_{2}=-0.1152 U_{1}+0.2455 \\
a_{3}=-0.1768 U_{1}+0.3097\end{array}$ \\
\hline $1.0964-1.0759$ & $\begin{array}{l}a_{1}=-0.1214 U_{1}+0.1897 \\
a_{2}=-0.1176 U_{1}+0.2305 \\
a_{3}=-0.2228 U_{1}+0.3647\end{array}$ & $0.5390-0.4566$ & $\begin{array}{c}a_{1}=-0.0435 U_{1}+0.1683 \\
a_{2}=-8.9980 \mathrm{e}-4 U_{1}+ \\
0.1792 \\
a_{3}=-0.0938 U_{1}+0.2615\end{array}$ \\
\hline $1.0761-1.0435$ & $\begin{array}{l}a_{1}=-0.1081 U_{1}+0.1753 \\
a_{2}=-0.0209 U_{1}+0.1265 \\
a_{3}=-0.1367 U_{1}+0.2721\end{array}$ & $0.4573-0.4563$ & $\begin{array}{l}a_{1}=-0.0594 U_{1}+0.1756 \\
a_{2}=-0.0218 U_{1}+0.1888 \\
a_{3}=-0.0983 U_{1}+0.2636\end{array}$ \\
\hline $1.0442-1.0215$ & $\begin{array}{l}a_{1}=-0.1268 U_{1}+0.1949 \\
a_{2}=-0.0605 U_{1}+0.1679 \\
a_{3}=-0.1531 U_{1}+0.2892\end{array}$ & $0.4562-0.3584$ & $\begin{array}{c}a_{1}=-0.0450 U_{1}+0.1690 \\
a_{2}=0.0106 U_{1}+0.1740 \\
a_{3}=-0.0799 U_{1}+0.2552\end{array}$ \\
\hline $1.0212-0.9777$ & $\begin{array}{l}a_{1}=-0.1128 U_{1}+0.1806 \\
a_{2}=-0.0041 U_{1}+0.1103 \\
a_{3}=-0.1081 U_{1}+0.2432\end{array}$ & $0.3585-0.2635$ & $\begin{array}{c}a_{1}=-0.0351 U_{1}+0.1655 \\
a_{2}=0.0311 U_{1}+0.1667 \\
a_{3}=-0.0690 U_{1}+0.2513\end{array}$ \\
\hline $0.9780-0.9529$ & $\begin{array}{l}a_{1}=-0.1416 U_{1}+0.2088 \\
a_{2}=-0.0499 U_{1}+0.1550 \\
a_{3}=-0.1215 U_{1}+0.2564\end{array}$ & $0.2641-0.2458$ & $\begin{array}{c}a_{1}=-0.0551 U_{1}+0.1708 \\
a_{2}=0.0086 U_{1}+0.1726 \\
a_{3}=-0.0713 U_{1}+0.2519\end{array}$ \\
\hline $0.9517-0.9185$ & $\begin{array}{l}a_{1}=-0.1254 U_{1}+0.1933 \\
a_{2}=-0.0085 U_{1}+0.1156 \\
a_{3}=-0.0937 U_{1}+0.2298\end{array}$ & $0.2453-0.1334$ & $\begin{array}{c}a_{1}=-0.0451 U_{1}+0.1683 \\
a_{2}=0.0253 U_{1}+0.1685 \\
a_{3}=-0.0645 U_{1}+0.2502\end{array}$ \\
\hline $0.9185-0.8943$ & $\begin{array}{l}a_{1}=-0.1720 U_{1}+0.2361 \\
a_{2}=-0.0698 U_{1}+0.1718 \\
a_{3}=-0.1055 U_{1}+0.2407\end{array}$ & $0.1347-0.1254$ & $\begin{array}{c}a_{1}=-0.0242 U_{1}+0.1655 \\
a_{2}=0.0451 U_{1}+0.1658 \\
a_{3}=-0.0655 U_{1}+0.2504\end{array}$ \\
\hline $0.8952-0.8762$ & $\begin{array}{l}a_{1}=-0.1592 U_{1}+0.2246 \\
a_{2}=-0.0656 U_{1}+0.1681 \\
a_{3}=-0.1134 U_{1}+0.2478\end{array}$ & $0.1246-0.0029$ & $\begin{array}{c}a_{1}=-0.0128 U_{1}+0.1641 \\
a_{2}=0.0593 U_{1}+0.1641 \\
a_{3}=-0.0627 U_{1}+0.2500\end{array}$ \\
\hline $0.8757-0.8709$ & $\begin{array}{l}a_{1}=-0.1444 U_{1}+0.2117 \\
a_{2}=-0.0297 U_{1}+0.1367 \\
a_{3}=-0.0896 U_{1}+0.2269\end{array}$ & - & $\begin{array}{l}- \\
- \\
-\end{array}$ \\
\hline
\end{tabular}

As is known, after the interval number $\mathrm{N}$ changes from 64 to 128 , the number of modulation segments increases dramatically. To get a lower modulation index for the switch angles, the precision of the switch angles is improved as well, but the calculation is increased with the corresponding computation.

\section{Simulation Result}

Based on the data in the above tables, this paper draws the change track of the angle of the harmonic elimination switch with the amplitude of the fundamental voltage, as shown in Figure 4. 


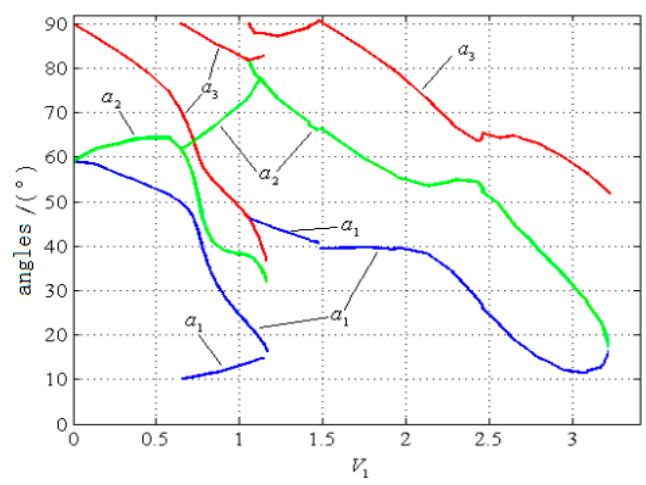

Figure 4. The trajectories of switch angles for each waveband.

It can be seen from Figure 4 that the correct switching angle can be obtained for each band of the modulation index in the range of $0-1$. Moreover, it can be found that there are multiple sets of switch angle trajectories in each cell near each switch band. These redundant switch angles can increase the flexibility of the switch angle selection and improve the control performance in the actual control.

To test and verify the correctness of the above theoretical analysis and the solving solution, MATLAB and SIMULINK software were used for the seven-level inverter of the multiband SHEPWM based on the Walsh function transform to build the model and do the experiment. The simulation parameters were as follows: the independent dc voltage source was $50 ; \mathrm{A}, \mathrm{B}$, and $\mathrm{C}$ bands took fundamental voltage amplitude $U_{1}$ as $3.15,1.32$, and 1.0, respectively, when choosing the switch angles. The output voltage waveforms and spectrum distribution are shown in Figures 5-7.

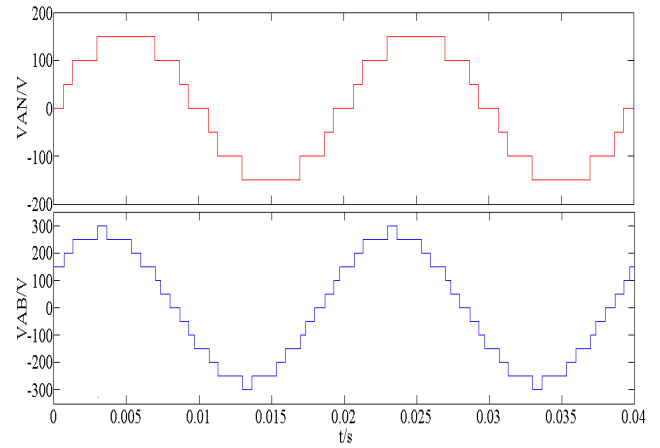

(a)

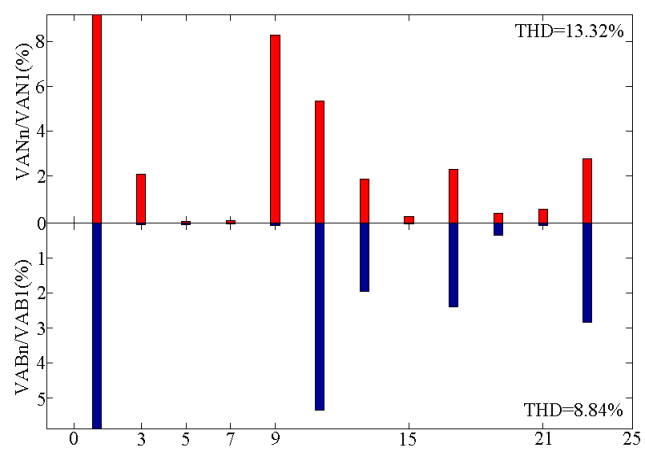

(b)

Figure 5. Simulation waveform based on $A$ waveband when $U_{1}=3.15$. (a) Simulation waveforms of phase and line voltages. (b) Spectrum distribution of phase and line voltages.

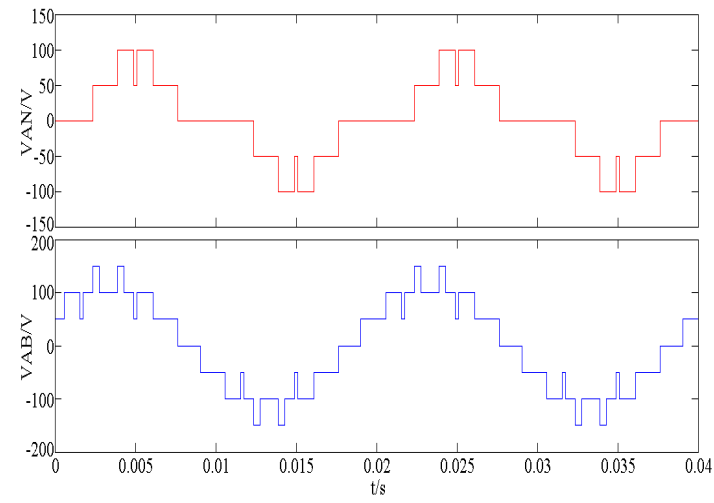

(a)

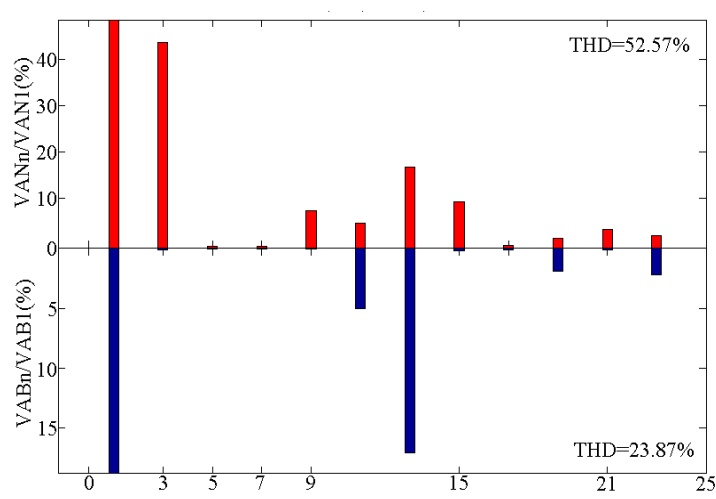

(b)

Figure 6. Simulation waveform based on the B waveband when $U_{1}=1.32$. (a) Simulation waveforms of phase and line voltages, (b) Spectrum distribution of phase and line voltages. 


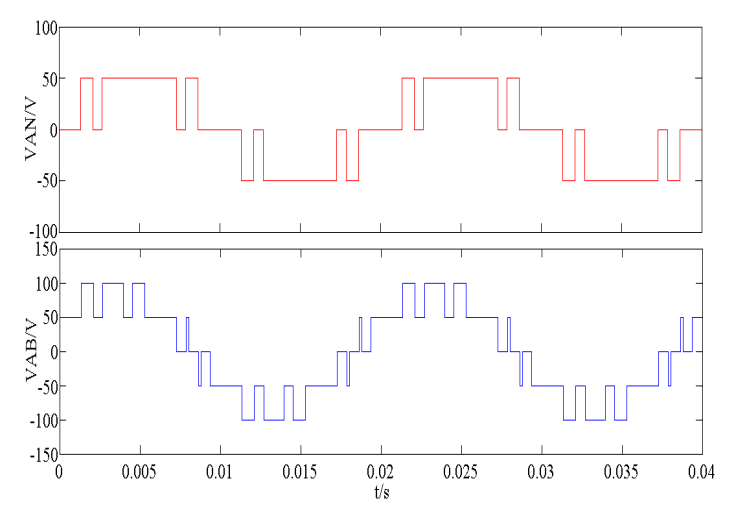

(a)

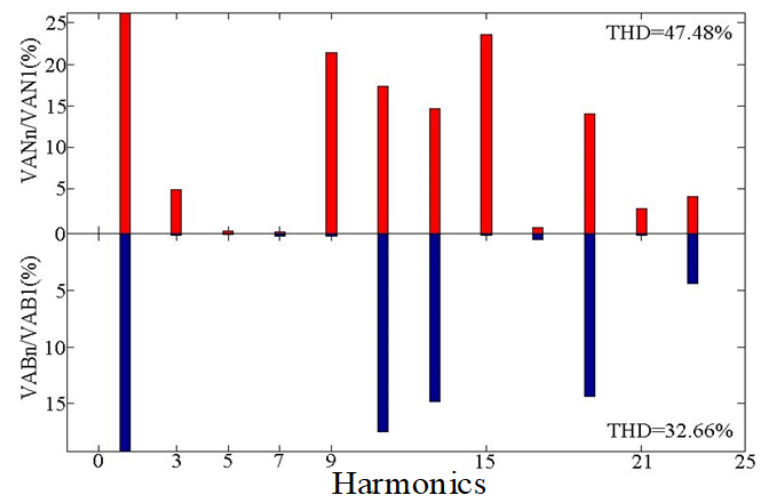

(b)

Figure 7. Simulation waveform based on the $C$ waveband when $U 1=1.0$. (a) Simulation waveforms of phase and line voltages. (b) Spectrum distribution of phase and line voltages.

Figure 5 shows the waveform of the output phase voltage $V_{A N}$ and line voltage $V_{A B}$ in the $A$ band when the fundamental voltage amplitude is 3.15, along with the Fast Fourier Transform (FFT) analysis.

Here, $V_{A N}$ is the $A$ phase voltage, $V_{A B}$ is the line voltage, $V_{A N i}$ is the $A$ phase voltage harmonic, $V_{A B i}$ is the $A$ phase line voltage harmonic, $V_{A N 1}$ is the fundamental wave of the $A$ phase, and $V_{A B i}$ is the fundamental wave of the line voltage between phase $A$ and phase $B$.

According to Figure $5 \mathrm{a}$, it can be seen that the phase voltage $\mathrm{V}_{\mathrm{AN}}$ is 7 levels and the line voltage $\mathrm{V}_{\mathrm{AB}}$ is 13 levels, which conforms to the design of the $\mathrm{A}$ band. From Figure $5 \mathrm{~b}$, it can be seen that the Total Harmonic Distortion (THD) of the phase voltage $\mathrm{V}_{\mathrm{AN}}$ is $13.32 \%$, in which the 5 th and 7 th harmonics are eliminated. The THD of the line voltage $\mathrm{V}_{\mathrm{AB}}$ is $8.84 \%$, in which the $3 \mathrm{rd}$, 5 th, and $7 \mathrm{th}$ harmonics are eliminated, which proves the correctness of the switch angle calculation. At the same time, a high-quality voltage waveform can be obtained under the SHEPWM modulation.

Figure 6 shows the waveform of the output phase voltage $V_{A N}$ and the line voltage $V_{A B}$ in the $B$ band when the fundamental voltage amplitude is 1.32, along with the FFT analysis.

According to Figure $6 \mathrm{a}$, it can be seen that the phase voltage $\mathrm{V}_{\mathrm{AN}}$ is 5 levels, while the line voltage $\mathrm{V}_{\mathrm{AB}}$ is 7 levels, which conforms to the design of the $\mathrm{B}$ band. From Figure $6 \mathrm{~b}$, it can be seen that the THD of the phase voltage $\mathrm{V}_{\mathrm{AN}}$ is $52.57 \%$, in which the 5 th and 7 th harmonics are eliminated. The THD of line voltage $\mathrm{V}_{\mathrm{AB}}$ is $23.87 \%$, in which the $3 \mathrm{rd}$, 5 th, and 7 th harmonics are eliminated, which proves the correctness of the switch angle calculation.

Figure 7 shows the waveform of the output phase voltage $V_{A N}$ and the line voltage $V_{A B}$ in the $C$ band when the fundamental voltage amplitude is 1.0, along with the FFT analysis.

As shown in the Figure $7 \mathrm{a}$, the phase voltage $V_{\mathrm{AN}}$ is 3 levels, while the line voltage $\mathrm{V}_{\mathrm{AB}}$ is 5 levels, which conforms to the design of the $C$ band. From Figure $7 \mathrm{~b}$, it can be seen that the THD of phase voltage $\mathrm{V}_{\mathrm{AN}}$ is $47.48 \%$, in which the 5 th and 7 thharmonics are eliminated. The THD of line voltage $\mathrm{V}_{\mathrm{AB}}$ is $32.66 \%$, in which the $3 \mathrm{rd}$, 5 th, and 7 th times harmonics are eliminated, which proves the correctness of the switch angle calculation.

As shown in Figures 5-7, the 5th and 7th harmonics have basically been eliminated, which can be proved using the control technology of the SHEPWM multilevel inverter based on the Walsh transform, combined with multiband modulation, which is feasible in this paper. Because of the limitation of the calculation accuracy and the precision of the numerical algorithm needed to solve the switch angles, the harmonic cannot be completely eliminated. Usually it will have a small amount of deviation, and the calculation of the accuracy of the switch angles under different modulation indexes is not the same.

In this paper, the effects on harmonic elimination under various modulations based on multilevel inverters have been studied, and 5th and 7th harmonic indexes have been listed. The amplitudes of 5th and 7th harmonics, which have been completely eliminated, theoretically are actually not zero, as 
shown in Figure 8a. The 5th harmonic indexes based on 64 and 128 phase voltage interval schemes under the $\mathrm{C}$ band model are given in Figure $8 \mathrm{~b}$.

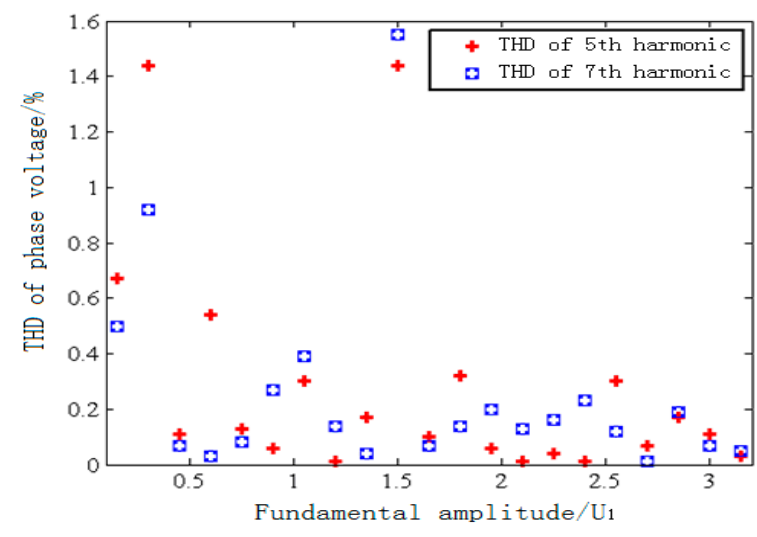

(a)

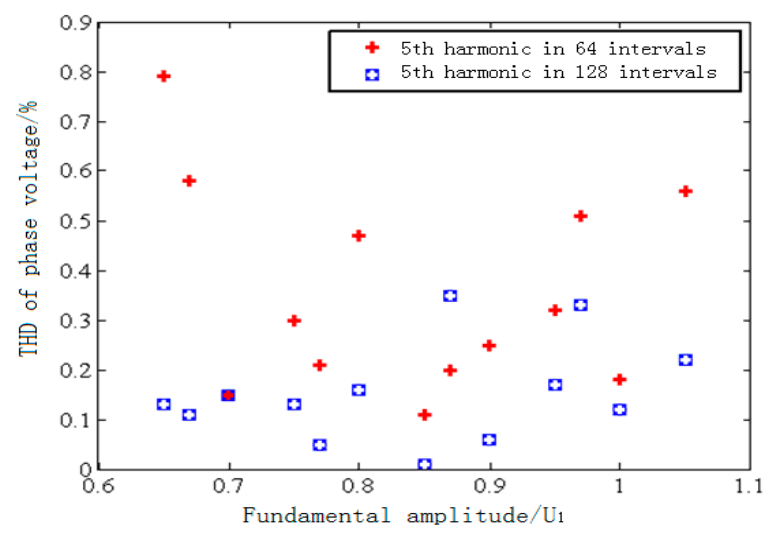

(b)

Figure 8. Harmonic percentage with fundamental amplitude in the phase voltage. (a) Percentage of harmonic with fundamental amplitude for 5th, 7th harmonic in phase voltage. (b) Percentage of harmonic with fundamental amplitude for 5 th harmonic based on $C$ waveband.

As shown in the Figure 8a, although the 5th and 7th harmonics have not been completely eliminated, there is a very small amount of error. The values are different under various modulation indexes. The errors indicate that the switch angles of the SHEPWM are not accurate, but they can be completely ignored in actual engineering control. From Figure $8 \mathrm{~b}$, the harmonic indexes in the 128-interval scheme are lower than the 64-interval scheme overall. This proves that the larger the interval scheme that is selected, the more precise the switch angels are and the better harmonic elimination effect it has.

\section{Experiment Verification}

In order to prove the feasibility of the algorithm proposed above and the harmonic elimination effect in the practical engineering, an inverter experiment platform is set up, whose parameters are reported in Table 8. An interface board is designed to do the isolated signal conditioning required for Analog to Digital Converter (ADC) module of a Digital Signal Processor(DSP)controller. The utilized processor is a TMS320F2812(Texas Instruments, Texas, USA) controller used to perform all necessary functions in the discrete time domain.

Table 8. Key parameters of the experiment.

\begin{tabular}{cc}
\hline Paremeter & Value \\
\hline Number of H-bridges & 3 \\
DC bus voltage & $22 \mathrm{~V}$ \\
Load & $40 \Omega$ \\
Output voltage frequency & $50 \mathrm{~Hz}$ \\
\hline
\end{tabular}

The output phase voltage $\mathrm{V}_{\mathrm{AN}}$ of the inverter under the SHEPWM modulation when the modulation index is 0.75 and its spectrum are shown in Figure $9 a, b$, respectively. Figure $9 c, d$ show the output phase voltage $\mathrm{V}_{\mathrm{AN}}$ of the inverter when the modulation is 0.46 and its spectrum, respectively. Figure $9 \mathrm{e}, \mathrm{f}$ show the output phase voltage $\mathrm{V}_{\mathrm{AN}}$ of the inverter when the modulation is 0.27 and its spectrum, respectively. 


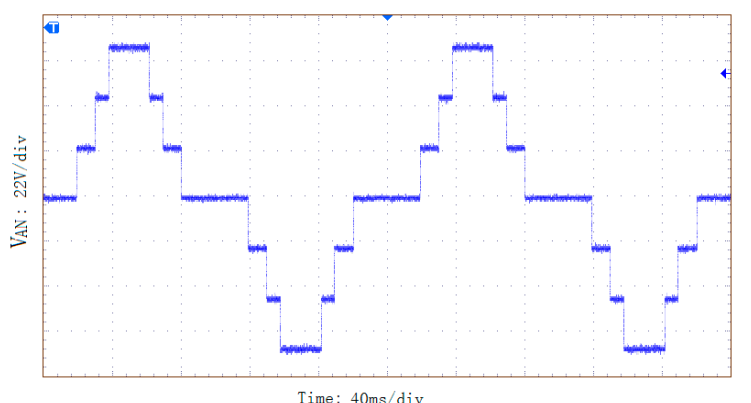

(a)

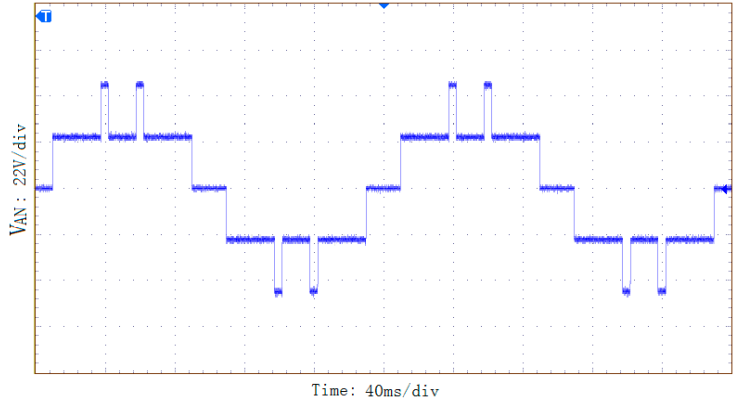

(c)

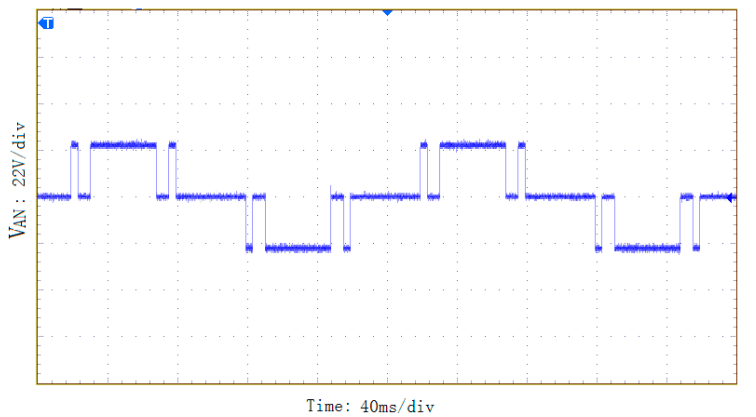

(e)

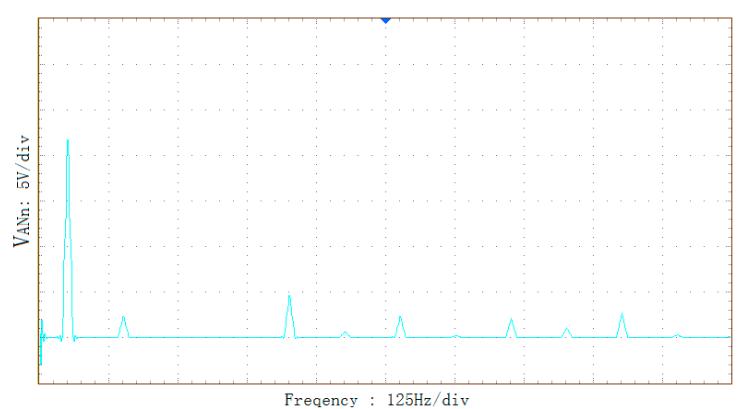

(b)

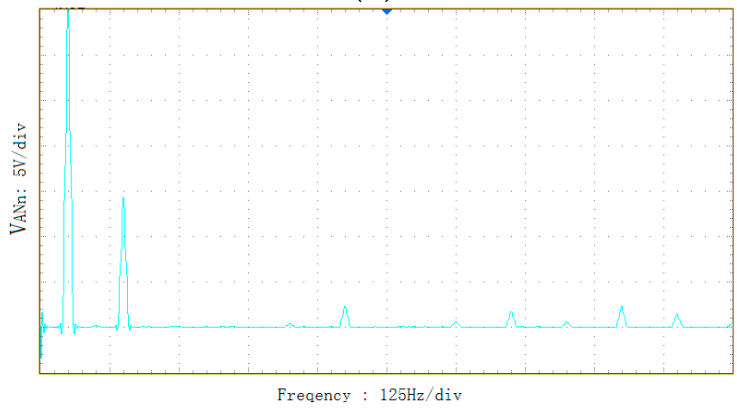

(d)

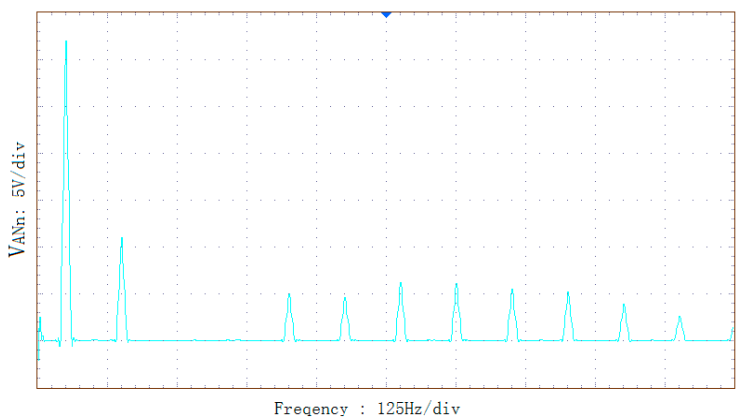

(f)

Figure 9. The phase voltage experiment waveforms and spectrums for A, B, and C patterns. (a) Phase voltage waveform for the A band. (b) Phase voltage spectrum for the A band. (c) Phase voltage waveform for the B band. (d) Phase voltage spectrum for the B band. (e) Phase voltage waveform for the $\mathrm{C}$ band. (f) Phase voltage spectrum for the $\mathrm{C}$ band.

When the modulation index is 0.75 , it corresponds to the SHEPWM modulation in the A band. Figure 9a shows that the output phase voltage is 7 levels. In addition, Figure $9 \mathrm{~b}$ shows that the 5 th and 7 th harmonics are basically eliminated and the THD of the phase voltage $\mathrm{V}_{\text {AN }}$ is $34.9 \%$.

When the modulation index is 0.46, it corresponds to the SHEPWM modulation in the B band. Figure $9 \mathrm{~b}$ shows that the output phase voltage is 5 levels. In addition, Figure 9e shows that the 5thand 7 th harmonics are basically eliminated and the THD of the phase voltage $\mathrm{V}_{\mathrm{AN}}$ is $43.1 \%$.

When the modulation index is 0.27, it corresponds to the SHEPWM modulation in the C band. Figure $9 \mathrm{c}$ shows the output phase voltage is 3 levels. In addition, Figure $9 \mathrm{f}$ shows that the 5 th and 7 th harmonics are basically eliminated and the THD of the phase voltage $\mathrm{V}_{\mathrm{AN}}$ is $63.2 \%$.

Overall, the correctness of the switch angle obtained by the multilevel inverter for the multiband SHEPWM control technology based on the Walsh function is proven. In addition, the multiband SHEPWM control technology of the multilevel inverter based on the Walsh function proposed in this paper can solve the problem of the limited modulation index in the specific harmonic elimination PWM technology effectively. 


\section{Conclusions}

The following conclusions can be summarized from the analysis and research for the multiband SHEPWM control method based on the Walsh function transform for the multilevel inverter.

1. By combining the Walsh function transformation with the multiband SHEPWM control method for the multilevel inverter, the regulation operation range of the inverter can be expanded to $0-1$. At the same time, the traditional SHEPWM nonlinear equations are transformed into piecewise linear equations, and the online calculation of the switching angle of the SHEPWM multilevel inverter is obtained, which makes real-time harmonic elimination control possible.

2. The switch angles cannot be solved with the fundamental current amplitude, which is below 0.656, by using 64-interval accuracy for the Walsh function transformation, but the switch angles under the lower precision modulation index can be solved by using the 128-interval Walsh function transformation.

3. The simulation results show that the 5 th and 7 th harmonics of each band modulation mode are nearly eliminated, which proves that the method based on the Walsh function is correct and feasible.

Author Contributions: Project management, M.Y.; conceptualization, G.S.; methodology, G.S. and S.L.; analysis, G.S.; software writing, Q.W.; validation, G.S., W.R., and S.L.; writing-original draft preparation, G.S.; writing-review and editing, M.Y. All authors have read and agreed to the published version of the manuscript.

Funding: This research was funded by National Natural Science Foundation of China, grant number 51767007; Jiangxi Provincial Industrial Science and Technology Support Project, grant number 20192BBEL50011; Jiangxi Natural Science Foundation Project, grant number 20192BAB206036; Jiangxi Provincial Youth Science Foundation of China, grant number GJJ190312.

Conflicts of Interest: There are no conflicts of interest regarding the publication of this paper.

\section{References}

1. Venkataramanaiah, J.; Suresh, Y.; Anup, K.P. Development of a New Hybrid Multilevel Inverter Using Modified Carrier SPWM Switching Strategy. IEEE Trans. Ind. Electron. 2018, 33, 8192-8197.

2. Ye, M.Y.; Nie, Y. Research on photovoltaic grid connected control of three-phase cascade H-bridge. J. East China Jiaotong Univ. 2019, 36, 99-104.

3. Saroj, K.S.; Tanmoy, B. Phase-Shifted carrier-based synchronized sinusoidal PWM techniques for a cascaded H-bridge multilevel inverter. IEEE Trans. Power Electron. 2018, 33, 513-524.

4. Edpuganti, A.; Rathore, A.K. Fundamental switching frequency optimal pulse width modulation of medium-voltage cascaded seven-level inverter. IEEE Trans. Ind. Appl. 2015, 51, 3485-3492. [CrossRef]

5. Sanjiba, K.B.; Nirmal, K.A. SHE-PWM technique for three phase three level voltage source inverter. In Proceedings of the 2017 IEEE International Conference on Power, Control, Signals and Instrumentation Engineering (ICPCSI), Chennai, India, 21-22 September 2017.

6. Ahmed, M.O.G.M. Implementation of SHE-PWM Technique for Parallel Voltage Source Inverters Employed in Uninterruptible Power Supplies. In Proceedings of the 2018 IEEE International Telecommunications Energy Conference (INTELEC), Turin, Italy, 7-11 October 2018.

7. Akshaya, D.B.; Prafullachandra, M.; Bedekar, P.P. Application of quarter-wave symmetry selective harmonic elimination PWM (SHE-PWM) to nested neutral point clamped (NNPC) converter. In Proceedings of the 2017 Innovations in Power and Advanced Computing Technologies (I-PACT), Vellore, India, 21-22 April 2017.

8. Yang, K.H.; Lu, D.Y.; Kuang, X.Q. Harmonic elimination for multilevel converters with unequal DC levels by using the polynomial homotopy continuation algorithm. In Proceedings of the 2016 35th Chinese Control Conference (CCC), Chengdu, China, 27-29 July 2016.

9. Jesus, V.; Rafael, P.; Inmaculada, M. Design guidelines using selective harmonic elimination advanced method for DC-AC PWM with the Walsh transform. In Proceedings of the 2011 7th International Conference-Workshop Compatibility and Power Electronics (CPE), Tallinn, Estonia, 1-3 June 2011. 
10. Zulfikar; Shuja, A.; Abbasi, A.R.M. A Novel Complete Set of Walsh and Inverse Walsh Transforms for Signal Processing. In Proceedings of the 2011 International Conference on Communication Systems and Network Technologies, Katra, India, 3-5 June 2011; IEEE Computer Society: Washington, DC, USA, 2011. [CrossRef]

11. Zhao, Y.L.; He, B.; Tong, J.Z. Application of selective harmonic elimination PWM technique based on walsh transform in DVR. Power Syst. Technol. 2006, 30, 45-49.

12. Zheng, C.F.; Zhang, B.; Qiu, D.Y. Solving switching angles for the inverter's selected harmonic elimination technique with Walsh function. In Proceedings of the 2005 International Conference on Electrical Machines and Systems, Nanjing, China, 27-29 September 2005.

13. Guan, E.Y.; Song, P.G.; Ye, M.Y. Selective harmonic elimination techniques for multilevel cascaded H-bridge inverters. In Proceedings of the 2005 International Conference on Power Electronics and Drives Systems, Kuala Lumpur, Malaysia, 28 November-1 December 2005.

14. Chiasson, J.N.; Tolbert, L.M.; McKenzie, K.J. A unified approach to solving the harmonic elimination equations in multilevel converters. IEEE Trans. Power Electron. 2004, 19, 478-490. [CrossRef]

15. Sai, L.; Liu, C.; Lu, F. A Reconstructing Method of PSWF Pulse Based on Three-valued Walsh Interpolation Functions. In Proceedings of the 2019 IEEE 3rd Information Technology, Networking, Electronic and Automation Control Conference (ITNEC), Chengdu, China, 15-17 March 2019.

16. Rosu, S.G.; Radoi, C.; Florescu, A.; Stocklosa, O. Microcontroller-based Walsh function PWM modulation with harmonic injection technique. In Proceedings of the 2010 IEEE International Conference on Automation, Quality and Testing, Robotics (AQTR), Cluj-Napoca, Romania, 28-30 May 2010.

(C) 2020 by the authors. Licensee MDPI, Basel, Switzerland. This article is an open access article distributed under the terms and conditions of the Creative Commons Attribution (CC BY) license (http://creativecommons.org/licenses/by/4.0/). 